\title{
Diurnal Variation of Melatonin Concentration in the Cerebrospinal Fluid of Unanesthetized Microminipig
}

\author{
MASAHARU ABE ${ }^{1}$, HIROAKI KAWAGUCHI ${ }^{1}$, NAOKI MIURA ${ }^{2}$, KOHEI AKIOKA ${ }^{3}$, MIHARU USHIKAI ${ }^{1}$, \\ SAYUMI OI ${ }^{4}$, AIRO YUKAWA ${ }^{4}$, TETSUYA YOSHIKAWA ${ }^{4}$, HIROYUKI IZUMI ${ }^{4}$ and MASAHISA HORIUCHI ${ }^{1}$ \\ ${ }^{1}$ Department of Hygiene and Health Promotion Medicine, \\ Kagoshima University Graduate School of Medical and Dental Sciences, Kagoshima, Japan; \\ ${ }^{2}$ Veterinary Teaching Hospital and ${ }^{3}$ Laboratory of Veterinary Histopathology, \\ Joint Faculty of Veterinary Medicine, Kagoshima University, Kagoshima, Japan; \\ ${ }^{4}$ Shin Nippon Biomedical Laboratories, Ltd., Kagoshima, Japan
}

\begin{abstract}
Background/Aim: The aim of this study was to develop a method for sequentially collecting cerebrospinal fluid (CSF) from an unanesthetized microminipig, which shares many physiological and anatomical similarities with humans, such as diurnality, and investigate the diurnal variation of melatonin concentration in the CSF. Materials and Methods: A catheter was placed percutaneously into the subarachnoid space of an anesthetized animal, and the tip of the catheter was placed into the cisterna magna under X-ray. We then sequentially collected CSF at lighton and -off times from the unanesthetized animal for several weeks. After catheter placement, a period of one week or more was necessary to relieve the contamination of RBCs in the CSF. Results: A higher melatonin level in the CSF was noted during lights-off time, and the level was higher than that in the serum. Conclusion: This model of sequential collection of CSF will contribute to research in brain functions.
\end{abstract}

Studies of cerebrospinal fluid (CSF) have been conducted in physiological and biochemical areas such as sleep and dementia (1). CSF collection methods have been reported for various animals. In recent years, there has been an increase in the number of studies on miniature pigs, which have many

This article is freely accessible online.

Correspondence to: Hiroaki Kawaguchi and Masahisa Horiuchi, Department of Hygiene and Health Promotion Medicine, Graduate School of Medical and Dental Sciences, Kagoshima University, 835-1 Sakuragaoka, Kagoshima 890-8544, Japan. Tel: +81 992755291, Fax: +81 992658434, e-mail: k3038952@kadai.jp (HK) masakun@m.kufm.kagoshima-u.ac.jp (MH)

Key Words: Intrathecal catheter, cerebrospinal fluid, melatonin, swine, minipig. similarities to humans such as diurnal, omnivorous, physiology, lipid metabolism and anatomy (2-6); however, there are few reports on porcine CSF collection methods. Development of a specific technique for sequential collection of CSF using transdermal intrathecal catheter in minipigs could represent a significant advance in both veterinary clinical anesthesia and analgesia, as well as in translational medicine in various areas of brain research such as sleep (7).

Intrathecal injection techniques using a single puncture of the spinal space have already been described in laboratory animals (8), including pigs (9); however, studies concerning the placement of a spinal catheter are limited to only a few animal models $(10,11)$. To the best of our knowledge, there are few studies describing the insertion of a spinal catheter in pigs $(6,12)$. To date, a less invasive insertion technique of CSF samples for porcine animal experimentation has not been developed (6).

In 2011, the microminipig was developed as the smallest miniature pig in the world (13) and this strain has similar profiles of general biochemistry and hematology (14-17) to domestic swine and humans. The utility of microminipigs as experimental animals has been demonstrated in research on atherosclerosis (18-21), pharmacological cardiovascular research $(22,23)$, sleep conditions $(24,25)$, coagulation activity (17), toxicological studies $(26,27)$, genomic sequencing (28), and developmental engineering (29). As well as other miniature pigs, microminipig represents a potentially appropriate experimental model, since their lipoprotein metabolism $(21,30)$, as well as their anatomy, physiology and feeding and sleep habits are similar to those of humans.

The aim of this study was to evaluate and validate the technique for sequential collection of CSF from cisterna magna using transdermal intrathecal catheter by lumbosacral puncture and to confirm a diurnal variation of melatonin in CSF of the microminipig under an unanesthetized condition. 


\section{Materials and Methods}

Animals and diet. Five microminipigs were obtained from a breeder (Fuji Micra Inc., Shizuoka, Japan) and maintained in a dedicated room with filtered air laminar flow at Kagoshima University. The room was maintained at a temperature of $24 \pm 3^{\circ} \mathrm{C}$ and a relative humidity of $50 \pm 20 \%$, with a 12 -h light/dark cycle. Tap water was available ad libitum, and the animals were provided with a commercial porcine diet (Horeborekobuta; Marubeni Nisshin Feed Inc., Tokyo, Japan). All protocols were approved by the Ethics Committees of Animal Care and Experimentation, Kagoshima University (MD16073) and Shin Nippon Biomedical Laboratories, Ltd., Drug Safety Research Laboratories (IACUC 703-065), which is fully accredited by AAALAC International. Finally, the research was performed according to the Institutional Guidelines for Animal Experiments and in compliance with the Japanese Act on Welfare and Management of Animals (Act No. 105 and Notification No. 6).

Preliminary computed tomography $(C T)$ and anatomical study confirming locations for needle and catheter insertion. Using another two 2-year old intact animals, one male and one female, (body weight: 17.0 and $19.0 \mathrm{~kg}$, respectively), the spinal needle was inserted at the lumbar 6 (L6) and L7 intervertebral space in the prone position under deep anesthesia with isoflurane inhalation by guiding with the X-ray fluoroscopy (VPX-200, Canon Medical Systems Corporation, Tochigi, Japan). After confirming the outflow of CSF, contrast medium (OYPALOMIN 370, Konica Minolta Japan Inc. Tokyo, Japan) was injected into the intrathecal space. For visualizing the contrast medium, Whole body CT (Aquilion TSX201A, Canon Medical Systems Corporation) scanning was performed. Additionally, three male animals two of which were approximately 10 months old (body weight: 5.4 and $6.4 \mathrm{~kg}$ ), and one that was approximately 4-year old (body weight: $24.6 \mathrm{~kg}$ ) were used. These animals were anesthetized and then sacrificed by bilateral axillary artery exsanguination for anatomical study.

Case 1 (Checking blood cells contamination in CSF). A 2-year old female animal (body weight: $18.8 \mathrm{~kg}$ ) was used. The transdermal intrathecal catheter placement was performed under deep anesthesia induced by isoflurane inhalation. In the prone position, a $21 \mathrm{G}$ $(0.8 \times 89 \mathrm{~mm})$ spinal needle was inserted at the lumbosacral space in a ventro-cranial orientation forming a $60^{\circ}$ angle (Figure $1 \mathrm{~A}$ and $\mathrm{B}$ ). After pulling out the inner needle and confirming the outflow of $\mathrm{CSF}$, a guide wire (Radifocus Guidewire $\mathrm{M}, 0.46 \mathrm{~mm}$ in diameter, length $150 \mathrm{~cm}$; Terumo Corporation, Tokyo, Japan) was inserted into the spinal needle, and the top of the wire was localized at the cisterna magna under X-ray (Figure 1C). A polyethylene catheter (1.22 mm in outside diameter, Hagitec Co., Ltd., Chiba, Japan) was inserted, and the opening of the catheter was localized at the cisterna magna using the guide wire. The catheter was filled with contrast medium and was confirmed that the opening of the catheter was located in the cisterna magna using X-ray (Figure 1D). The catheter was fixed through the subcutaneous tissue of the back. Approximately $1 \mathrm{ml}$ samples of CSF leakage were collected from an unanesthetized animal every week for 3 weeks for observation of red blood cell contamination in CSF. The gross color and appearance of CSF were observed, and CSF cell counts such as red blood cell (RBC) and white blood cell (WBC) were performed using a Bürker-Türk hemocytometer method. CSF measurement for total protein (TP), lactate dehydrogenase $(\mathrm{LDH})$, creatine kinase $(\mathrm{CK})$, glucose $(\mathrm{Glc})$, sodium $(\mathrm{Na})$, potassium $(\mathrm{K})$, chloride $(\mathrm{Cl})$, and calcium (Ca) was carried out by Clinical Pathology Laboratory, Co. Ltd. (Kagoshima, Japan).

Case 2 (Study on sequential collection of CSF and diurnal variation of melatonin concentration in CSF). A 2-year-old female animal (body weight: $19.4 \mathrm{~kg}$ ) was used. The transdermal intrathecal catheter placement was performed in the same manner as described in Case 1. At the same time, a temperature logger (Thermochron, KN Laboratories, Tokyo Japan) for body temperature (BT) monitoring was implanted in the cervical subcutaneous region, and a harness with an actigraph (Octagonal Basic Motionlogger; Ambulatory Monitoring, Inc., Ardsley, NY, USA) for locomotor activity (LA) monitoring was attached $(24,25)$. BT and LA were measured for 24 hours during the test period. At 3 weeks after catheter placement, approximately $1 \mathrm{ml}$ CSF leakage was collected from an unanesthetized animal at 9:00, 15:00, and 21:00 on Day 1 and Day 3 for measurement of melatonin in addition to the seven parameters in Case 1 (Clinical Pathology Laboratory, Co. Ltd.). Blood was collected from the cranial vena cava at the same time as CSF collection and was analyzed in the same manner as CSF. Amino acids in the CSF and plasma including glutamine (Gln) and alanine (Ala) were also measured by high performance liquid chromatography (JLC-500/V, JEOL Ltd., Tokyo, Japan).

\section{Results}

In a preliminary study of CT imaging of the intrathecal space with contrast medium, we confirmed that the cisterna magna was wider than other parts at the intrathecal space of the cervical spine, and the rich contrast medium in the intrathecal space was recognized at the lumbosacral space while the relatively thin spinal cord appeared (Figure 2A). The last lumbar and first sacral (S1) intervertebral space are most reliable for the introduction of a needle since the cauda equina is anatomically located at the last lumber-S1 (Figure 2B and C).

The technique applied in Cases 1 and 2, allowed transdermal intrathecal catheter placement since the top of the catheter reached the cisterna magna in both animals, as confirmed by radiographic examinations. Neither animal showed abnormal conditions such as brief ataxia, and trouble with CSF leakage such as stenosis of the spinal canal or looping of the device itself were not noted during several weeks after recovery from anesthesia.

In Case 1, we confirmed the offset period after transdermal intrathecal catheter placement (Table I). The CSF just after catheter insertion was slightly reddish and slightly semitransparent, and the gross color changes of the CSF continued after 3 days. After 1 week, the CSF became colorless and transparent, but RBC count and LDH activity in the CSF were still high. These values decreased in 2-3 weeks. Neither a high or low level was observed in the other 6 biochemical parameters from 1 to 3 weeks after transdermal intrathecal catheter placement. 

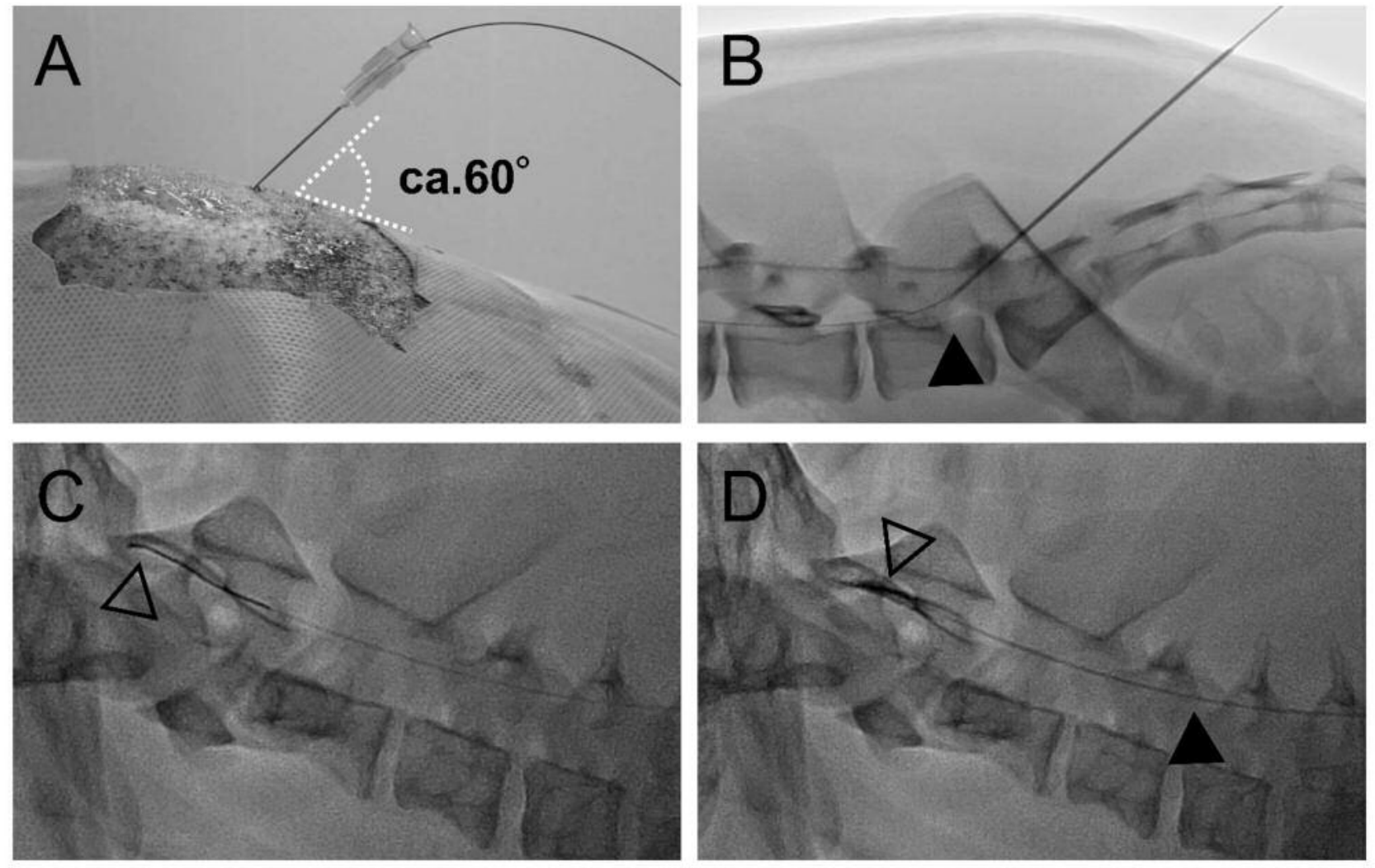

Figure 1. Transdermal intrathecal catheter by lumbosacral puncture in Cases 1 and $2(A-D)$. In the prone position, a spinal needle was inserted at the lumbosacral space in a ventro-cranial orientation forming an angle of approximately $60^{\circ}$ (A). The top of the needle was recognized by the closed triangle while it was inserted $(B)$. After pulling out the inner needle and confirming CSF leakage, the guide wire was inserted into the spinal needle, and the top of the wire was localized at the cisterna magna under X-ray (opened triangle, $C$ ). A polyethylene catheter was inserted using the guide wire. The opening of the catheter, which was filled with contrast medium (closed triangle, D), was recognized in the cisterna magna under X-ray (opened triangle, $D$ ).

In Case 2, we confirmed a technique for sequential collection of CSF to measure diurnal variation of melatonin and amino acids including Gln and Ala in CSF for confirming whether physiological variation of substances in CSF can be evaluated with this collection method (Table II). Melatonin in CSF showed a higher value at night than during the day time. However, no diurnal variation was observed in Gln or Ala. We also observed a decrease in BT and LA at night, representing the diurnality of microminipigs (Figure 3). Increases in BT and LA even at night were accordant with the collection of CSF and blood. In the same biochemical test as Case 1, LDH elevation was not observed (Table III).

\section{Discussion}

The technique described in this study allowed spinal catheter placement in all animals. The catheter slid in easily, proving the size of the device to be appropriate for such minipigs.
The preliminary study helped in finding adequate locations for needle and catheter insertion. In domestic piglets, the L2 and L3 intervertebral space was chosen as the most reliable and easily punctured, and a spinal needle was introduced between the L2 and L3 spinous processes in a ventro-cranial orientation until CSF leakage (6). The CT imaging with contrast medium of the intrathecal space revealed that the thick spinal cord disappeared at the intrathecal space of the last lumbar. We also confirmed the anatomical location of the cauda equina at the last lumberS1 in Microminipigs. Therefore, we considered the last lumbar and S1 intervertebral space to be most reliable for the introduction of the needle in prevention of trauma and hematoma in the spinal cord, because the spinal nerve of the cauda equina is thinner and minimally invasive. Moreover, since the sacrum has no spinous processes, it is easier to reach the intervertebral space in the prone position by approaching in the midline. The intrathecal space in the cisterna magna was wider than in other areas, suggesting that 

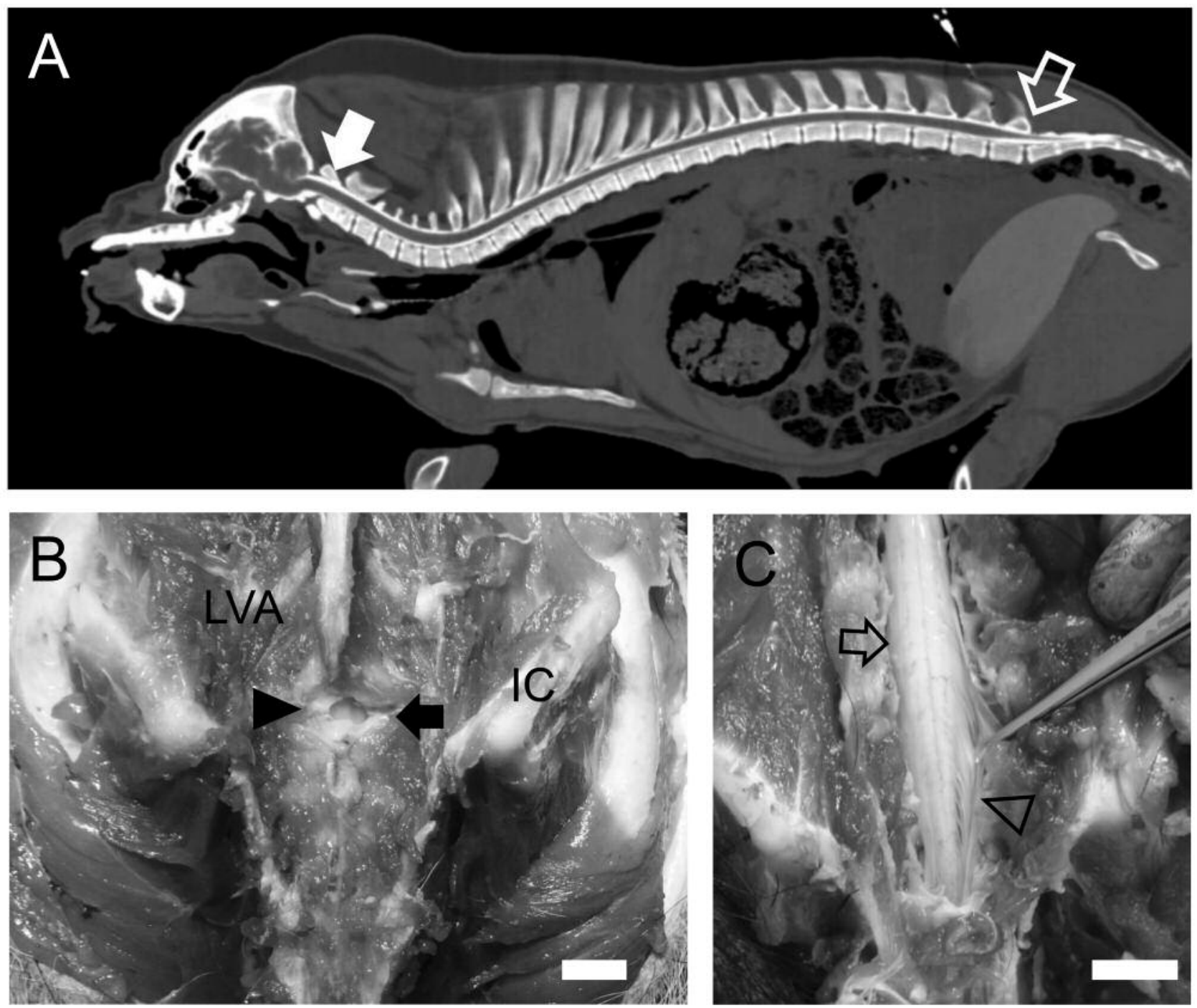

Figure 2. The preliminary study: Computed tomography image of the intrathecal space of cisterna magna (arrow) with intrathecal contrast medium was wider than the other areas of the intrathecal space of the cervical spine (A). The rich contrast medium in the intrathecal space was recognized at the lumbosacral space while the relatively thin spinal cord appeared (opened arrow), which was near the point of needle insertion. Lumbosacral space (closed arrow) is covered by ligamentum flavum (closed arrow head) and has enough space to insert the spinal needle (B). Lumbar enlargement (open arrow) is located on the head side than at the last lumber vertebra and the spinal cord at the lumbosacral space reveals cauda equina (open arrow head) (C). LVA: Lumbar vertebral arch; IC: iliac crest. Bar=1 cm.

there is more volume of CSF in this area. Moreover, neurotransmitter metabolite concentrations in CSF from the cisterna magna were higher than those in lumbar spinal fluid $(31,32)$. We decided to introduce the tip of the catheter into the cisterna magna.

During Cases 1 and 2, the catheter placement method using a guide wire was considered to be less invasive during puncture because the spinal needle was thin, and the inside diameter of the catheter could conversely be increased, thus a long-term patency period can be obtained. Based on the results in Table I, the method in this study does not affect the general condition of catheter indwelling for several weeks, and we also succeeded in performing sequential collection of CSF from an unanesthetized animal. Sequential collections of approximately $1 \mathrm{ml}$ of CSF leakage for several weeks was thought to be possible since the tip of the catheter was localized at the cisterna magna, which holds relatively rich CSF.

In Case 1, the offset period after transdermal intrathecal catheter placement required over 1 week to reduce the effects of erythrocyte contamination, because the colorless and 

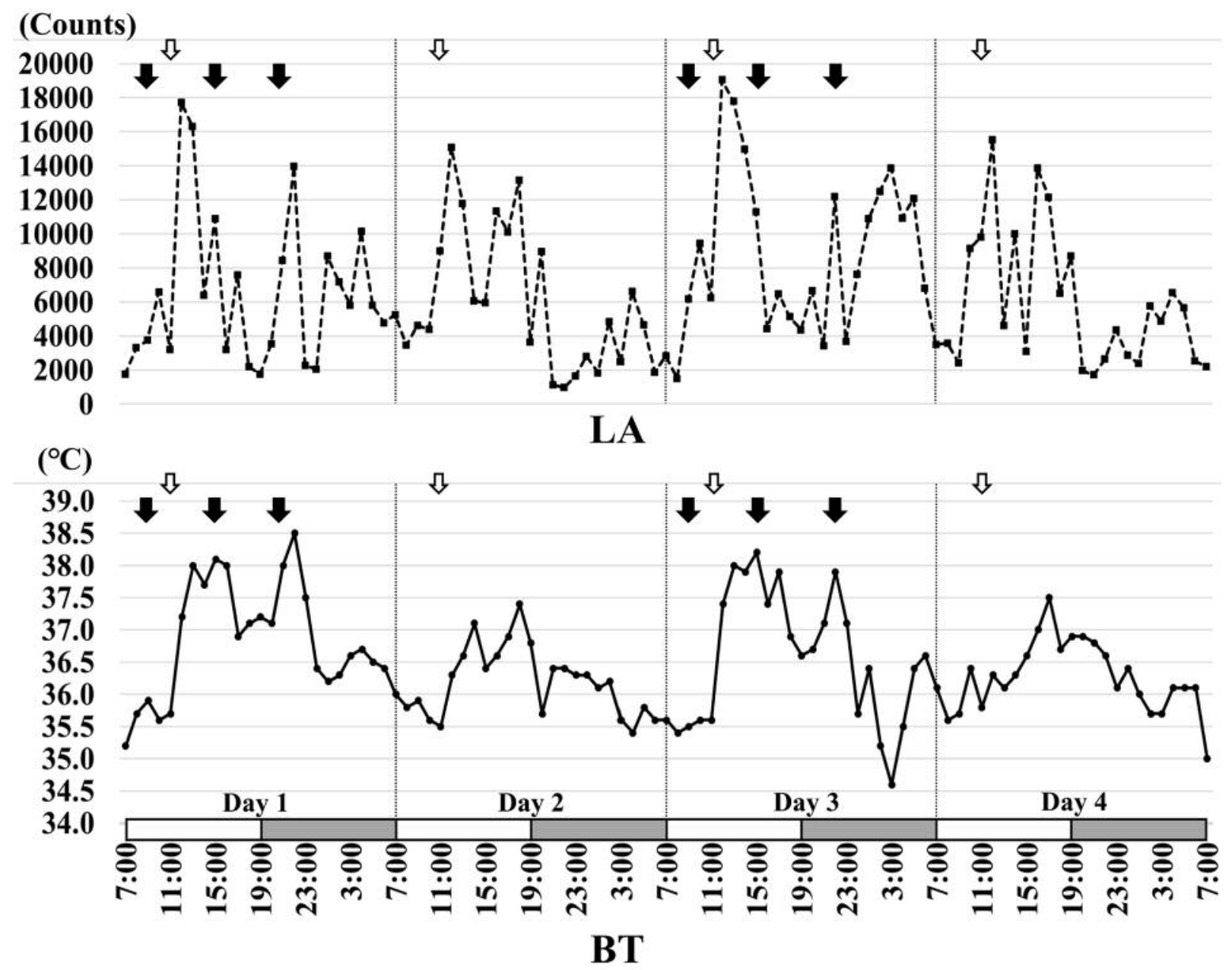

Figure 3. Locomotor activity (LA) and body temperature (BT) in Case 2: White and gray horizontal bands indicate the times that lights were on $(7: 00 \rightarrow 19: 00)$ and off $(19: 00 \rightarrow$ 7:00). Open and closed arrows indicate meal times and CSF and blood collection points. The left longitudinal axis indicates activity counts per hour by LA. LA and BT rise at feeding and collection, while the LA and BT during the night indicate comparatively low activity count and temperature.

Table I. Gross color and appearance, red blood cell count and chemistry of CSF in Case 1.

\begin{tabular}{|c|c|c|c|c|c|c|}
\hline & & At catheter insertion & Day 3 & Day 7 & Day 14 & Day 21 \\
\hline Gross color & & Slightly red & Slightly red & Colorless & Colorless & Colorless \\
\hline Gross appearance & & Slightly semitransparent & Slightly semitransparent & Transparent & Transparent & Transparent \\
\hline RBC & $\operatorname{cell} / \mu 1$ & $\mathrm{NE}$ & $\mathrm{NE}$ & 602.5 & 22.5 & 0.0 \\
\hline WBC & cell/ $/ \mu 1$ & $\mathrm{NE}$ & $\mathrm{NE}$ & 1120.0 & 102.5 & 2.5 \\
\hline Biochemical findings & & $\mathrm{NE}$ & $\mathrm{NE}$ & & & \\
\hline $\mathrm{TP}$ & $\mathrm{mg} / \mathrm{dl}$ & & & 77.8 & 50.3 & 45.3 \\
\hline LDH & $\mathrm{IU} / 1$ & & & 154.0 & 41.0 & 40.0 \\
\hline CK & $\mathrm{IU} / 1$ & & & 21.0 & 15.0 & 10.0 \\
\hline Glc & $\mathrm{mg} / \mathrm{dl}$ & & & 37.0 & 43.0 & 42.0 \\
\hline $\mathrm{Na}$ & $\mathrm{mEq} / 1$ & & & 148.0 & 149.0 & 150.0 \\
\hline $\mathrm{K}$ & $\mathrm{mEq} / \mathrm{l}$ & & & 3.6 & 3.3 & 3.3 \\
\hline $\mathrm{Cl}$ & $\mathrm{mEq} / \mathrm{l}$ & & & 121.0 & 120.0 & 123.0 \\
\hline $\mathrm{Ca}$ & $\mathrm{mg} / \mathrm{dl}$ & & & 5.6 & 5.3 & 5.4 \\
\hline
\end{tabular}

NE: Not examined; TP: total protein; LDH: lactate dehydrogenase; CK: creatine kinase; Glc: glucose; Na: sodium; K: potassium; Cl: chloride; Ca: calcium. 
in vivo $32: 583-590(2018)$

Table II. Diurnal variation of melatonin and amino acids concentration in CSF and blood in Case 2.

\begin{tabular}{|c|c|c|c|c|c|c|c|c|c|c|c|c|}
\hline & \multicolumn{6}{|c|}{ Day 1} & \multicolumn{6}{|c|}{ Day 3} \\
\hline & \multicolumn{2}{|c|}{ 9:00 } & \multicolumn{2}{|c|}{$15: 00$} & \multicolumn{2}{|c|}{ 21:00 } & \multicolumn{2}{|c|}{ 9:00 } & \multicolumn{2}{|c|}{$15: 00$} & \multicolumn{2}{|c|}{ 21:00 } \\
\hline & $\mathrm{CSF}$ & Blood & CSF & Blood & $\mathrm{CSF}$ & Blood & CSF & Blood & CSF & Blood & $\mathrm{CSF}$ & Blood \\
\hline Melatonin (pmol/l) & 47.4 & 99.0 & $<12.1$ & 38.3 & 290.6 & 99.0 & $<12.1$ & 56.0 & $<12.1$ & 12.5 & 129.2 & 51.7 \\
\hline $\operatorname{Gln}(\mu \mathrm{mol} / 1)$ & 588.7 & 331.7 & 452.7 & 426.9 & 543.7 & 352.0 & 453.9 & 341.3 & 386.0 & 376.4 & 448.7 & 397.4 \\
\hline Ala $(\mu \mathrm{mol} / \mathrm{l})$ & 152.0 & 437.6 & 144.8 & 530.9 & 111.3 & 480.2 & 144.5 & 453.4 & 168.6 & 603.1 & 133.5 & 515.3 \\
\hline
\end{tabular}

Gln: Glutamine; Ala: alanine.

Table III. Diurnal variation of CSF chemistry in Case 2.

\begin{tabular}{|c|c|c|c|c|c|c|c|}
\hline & & \multicolumn{3}{|c|}{ Day 1} & \multicolumn{3}{|c|}{ Day 3} \\
\hline & & 9:00 & $15: 00$ & 21:00 & 9:00 & $15: 00$ & 21:00 \\
\hline LDH & $\mathrm{U} / 1$ & 12.5 & 20.0 & 15.0 & 10.0 & 10.0 & 10.0 \\
\hline CK & $\mathrm{U} / 1$ & 2.5 & 2.5 & 2.5 & 5.0 & 2.5 & 2.5 \\
\hline Glc & $\mathrm{mg} / \mathrm{dl}$ & 29.0 & 28.0 & 23.0 & 28.0 & 31.0 & 24.0 \\
\hline $\mathrm{Na}$ & $\mathrm{mEq} / \mathrm{l}$ & 147.5 & 150.0 & 147.5 & 150.0 & 147.5 & 147.5 \\
\hline $\mathrm{K}$ & $\mathrm{mEq} / 1$ & 3.3 & 3.3 & 3.0 & 3.0 & 3.3 & 3.0 \\
\hline $\mathrm{Cl}$ & $\mathrm{mEq} / \mathrm{l}$ & 115.0 & 110.0 & 112.5 & 110.0 & 112.5 & 110.0 \\
\hline $\mathrm{Ca}$ & $\mathrm{mg} / \mathrm{dl}$ & 4.8 & 5.0 & 4.8 & 4.8 & 5.0 & 4.8 \\
\hline
\end{tabular}

LDH: Lactate dehydrogenase; CK: creatine kinase; Glc: glucose; Na: sodium; K: potassium; Cl: chloride; Ca: calcium.

transparent CSF at 1 week after the catheter placement revealed high $\mathrm{RBC}$ and $\mathrm{LDH}$.

In Case 2, the cisterna magna had relatively abundant CSF, and CSF collection was shown to be possible even more than once a day with no problems in the general condition of the animals. In addition, the concentrations of melatonin in the blood and CSF were high at light-off time like in humans (33), while no remarkable diurnal variation was observed in Gln or Ala. Moreover, the concentrations of melatonin in CSF at night were higher than those in blood, suggesting that melatonin is produced by the pineal gland, and there is a route directly from the pineal gland to CSF in addition to the route through blood circulation (34). We also confirmed that this animal was in a sleep-like state at night due to decreases in LA and BT at light-off time as shown in our previous report $(24,25)$. The increases in $\mathrm{LA}$ and $\mathrm{BT}$ even at night were accordant with the collection of CSF and blood, suggesting that the treatment disrupts transiently physiological sleep. Therefore, the collection of CSF at night should be conducted quickly.

In conclusion, we established a transdermal intrathecal catheter placement technique using microminipigs. This transdermal intrathecal catheter placement makes it possible to sequentially collect CSF, which shows the diurnal variation of melatonin, from the cisterna magna in an unanesthetized animal for several weeks. This microminipig model of sequential CSF collection contributes to physiological and biochemical research. Moreover, this technique may be useful in pharmacological research to examine CSF using sequential collections after administration of medicine.

\section{Conflicts of Interest}

There exist no conflicts of interest in regard to this study.

\section{Acknowledgements}

The Authors thank all the staff members of the Division of Laboratory Animal Sciences, Natural Science Center for Research and Education, Kagoshima University, who kept the animals in good condition. The Authors would also like to thank Dr. Kaichiro Takeishi for technical assistance and Ms. Jenn Fukuyama, Mr. Ben Hollingum and Ms. Connie Sinks from Shin Nippon Biomedical Laboratories, Ltd. for editing in this study. This research was partly supported by the Japan Society for the Promotion of Science (JSPS) Kakenhi [grant number 16K08023, HK] and the Kodama Memorial Fund for Medical Research (HK). 


\section{References}

1 Slats D, Claassen JA, Lammers GJ, Melis RJ, Verbeek MM and Overeem S: Association between hypocretin-1 and amyloidbeta42 cerebrospinal fluid levels in Alzheimer's disease and healthy controls. Curr Alzheimer Res 9: 1119-1125, 2012.

2 Bollen PJA, Hansen AK and Alstrup AKO: Important biological features. In: The Laboratory SWINE, 2nd edition. Bollen PJA, Hansen AK and Alstrup AKO (eds.). Northwest USA, CRC Press, pp. 1-13, 2010.

3 Swanson KS, Mazur MJ, Vashisht K, Rund LA, Beever JE, Counter CM and Schook LB: Genomics and clinical medicine: rationale for creating and effectively evaluating animal models. Exp Biol Med (Maywood) 229: 866-875, 2004.

4 Karali M, Manfredi A, Puppo A, Marrocco E, Gargiulo A, Allocca M, Corte MD, Rossi S, Giunti M, Bacci ML, Simonelli F, Surace EM, Banfi S and Auricchio A: MicroRNA-restricted transgene expression in the retina. PLoS One 6: e22166, 2011.

5 Testa F, Surace EM, Rossi S, Marrocco E, Gargiulo A, Di lorio V, Ziviello C, Nesti A, Fecarotta S, Bacci ML, Giunti M, Della Corte M, Banfi S, Auricchio A and Simonelli F: Evaluation of Italian patients with leber congenital amaurosis due to AIPL1 mutations highlights the potential applicability of gene therapy Invest Ophthalmol Vis Sci 52: 5618-5624, 2011.

6 Lambertini C, Ventrella D, Barone F, Sorrentino NC, Dondi F, Fraldi A, Giunti M, Surace EM, Bacci ML and Romagnoli N: Transdermal spinal catheter placement in piglets: Description and validation of the technique. J Neurosci Methods 255: 17-21, 2015 .

7 Lucey BP, Gonzales C, Das U, Li J, Siemers ER, Slemmon JR, Bateman RJ, Huang Y, Fox GB, Claassen JA, Slats D, Verbeek MM, Tong G, Soares H, Savage MJ, Kennedy M, Forman M, Sjogren M, Margolin R, Chen X, Farlow MR, Dean RA and Waring JF: An integrated multi-study analysis of intra-subject variability in cerebrospinal fluid amyloid-beta concentrations collected by lumbar puncture and indwelling lumbar catheter. Alzheimers Res Ther 7: 53, 2015.

8 Bevan AK, Duque S, Foust KD, Morales PR, Braun L, Schmelzer L, Chan CM, McCrate M, Chicoine LG, Coley BD, Porensky PN, Kolb SJ, Mendell JR, Burghes AH and Kaspar BK: Systemic gene delivery in large species for targeting spinal cord, brain, and peripheral tissues for pediatric disorders. Mol Ther 19: 1971-1980, 2011.

9 Romagnoli N, Ventrella D, Giunti M, Dondi F, Sorrentino NC, Fraldi A, Surace EM and Bacci ML: Access to cerebrospinal fluid in piglets via the cisterna magna: optimization and description of the technique. Lab Anim 48: 345-348, 2014.

10 Fairbanks CA: Spinal delivery of analgesics in experimental models of pain and analgesia. Adv Drug Deliv Rev 55: 10071041, 2003

11 Poon YY, Chang AY, Ko SF and Chan SH: Catheterization of the thoracic spinal subarachnoid space in mice. J Neurosci Methods 200: 36-40, 2011

12 Federici T, Taub JS, Baum GR, Gray SJ, Grieger JC, Matthews KA, Handy CR, Passini MA, Samulski RJ and Boulis NM Robust spinal motor neuron transduction following intrathecal delivery of AAV9 in pigs. Gene Ther 19: 852-859, 2012.

13 Kaneko N, Itoh K, Sugiyama A and Izumi Y: Microminipig, a non-rodent experimental animal optimized for life science research: preface. J Pharmacol Sci 115: 112-114, 2011.
14 Kawaguchi H, Yamada T, Miura N, Takahashi Y, Yoshikawa T, Izumi $\mathrm{H}$, Kawarasaki T, Miyoshi $\mathrm{N}$ and Tanimoto A: Reference values of hematological and biochemical parameters for the world smallest microminipigs. J Vet Med Sci 74: 933-936, 2012.

15 Kawaguchi $H$, Yamada $T$, Miura N, Noguchi M, Izumi $H$, Miyoshi $\mathrm{N}$ and Tanimoto A: Sex differences of serum lipid profile in novel microminipigs. In Vivo 27: 617-621, 2013.

16 Kakimoto T, Otsuka A, Kawaguchi H, Ogata K, Tanimoto A and Kanouchi H: Plasma homocysteine concentrations in novel microminipigs. In Vivo 28: 579-82, 2014.

17 Miura N, Kucho K, Noguchi M, Miyoshi N, Uchiumi T, Kawaguchi $\mathrm{H}$ and Tanimoto A: Comparison of the genomic sequence of the microminipig, a novel breed of swine, with the genomic database for conventional pig. In Vivo 28: 1107-1111, 2014.

18 Miyoshi N, Horiuchi M, Inokuchi Y, Miyamoto Y, Miura N, Tokunaga S, Fujiki M, Izumi Y, Miyajima H, Nagata R, Misumi $\mathrm{K}$, Takeuchi $\mathrm{T}$, Tanimoto $\mathrm{A}$, Yasuda $\mathrm{N}$, Yoshida $\mathrm{H}$ and Kawaguchi H: Novel microminipig model of atherosclerosis by high fat and high cholesterol diet, established in Japan. In Vivo 24: 671-680, 2010.

19 Akioka K, Kawaguchi H, Kitajima S, Miura N, Noguchi M, Horiuchi M, Miyoshi $\mathrm{N}$ and Tanimoto A: Investigation of necessity of sodium cholate and minimal required amount of cholesterol for dietary induction of atherosclerosis in microminipigs. In Vivo 28: 81-90, 2014.

20 Kawaguchi H, Miyoshi N, Miura N, Fujiki M, Horiuchi M, Izumi Y, Miyajima H, Nagata R, Misumi K, Takeuchi T, Tanimoto $\mathrm{A}$ and Yoshida $\mathrm{H}$ : Microminipig, a non-rodent experimental animal optimized for life science research: novel atherosclerosis model induced by high fat and cholesterol diet. J Pharmacol Sci 115: 115-121, 2011.

21 Kawaguchi H, Yamada T, Miura N, Ayaori M, Uto-Kondo H, Ikegawa M, Noguchi M, Wang KY, Izumi H and Tanimoto A: Rapid development of atherosclerosis in the world's smallest Microminipig fed a high-fat/high-cholesterol diet. J Atheroscler Thromb 21: 186-203, 2014

22 Yokoyama H, Nakamura Y, Saito H, Nagayama Y, Hoshiai K, Wada T, Izumi-Nakaseko H, Ando K, Akie Y and Sugiyama A: Pharmacological characterization of microminipig as a model to assess the drug-induced cardiovascular responses for non-clinical toxicity and/or safety pharmacology studies. J Toxicol Sci 42 : 93-101, 2017.

23 Zahorul Islam M, Kawaguchi H, Miura N, Miyoshi N, Yamazaki-Himeno E, Shiraishi M, Miyamoto A and Tanimoto A: Hypertension alters the endothelial-dependent biphasic response of bradykinin in isolated Microminipig basilar artery. Microvasc Res 114: 52-57, 2017.

24 Takeishi K, Horiuchi M, Kawaguchi H, Deguchi Y, Izumi H, Arimura E, Kuchiiwa S, Tanimoto $\mathrm{A}$ and Takeuchi $\mathrm{T}$ : acupuncture improves sleep conditions of minipigs representing diurnal animals through an anatomically similar point to the acupoint (GV20) effective for humans. Evid Based Complement Alternat Med 2012: 472982, 2012.

25 Takeishi K, Kawaguchi H, Akioka K, Noguchi M, Arimura E, Abe M, Ushikai M, Okita S, Tanimoto A and Horiuchi M: Effects of dietary and lighting conditions on diurnal locomotor activity and body temperature in microminipigs. In Vivo 32: 55$62,2018$. 
26 Yoshikawa T, Takahashi Y, Kawaguchi H, Utsunomiya S, Miura $\mathrm{N}$, Izumi $\mathrm{H}$, Miyoshi $\mathrm{N}$ and Tanimoto A: A dermal phototoxicity study following intravenous infusion administration of ciprofloxacin hydrochloride in the novel microminipigs. Toxicol Pathol 41: 109-113, 2013.

27 Guruge KS, Noguchi M, Yoshioka K, Yamazaki E, Taniyasu S, Yoshioka M, Yamanaka N, Ikezawa M, Tanimura N, Sato M, Yamashita $\mathrm{N}$ and Kawaguchi $\mathrm{H}$ : Microminipigs as a new experimental animal model for toxicological studies: comparative pharmacokinetics of perfluoroalkyl acids. J Appl Toxicol 36: 6875, 2016.

28 Miura N, Kawaguchi H, Nagasato T, Yamada T, Ito T, Izumi H, Shameshima H, Miyoshi N, Tanimoto A and Maruyama I: Coagulation activity and white thrombus formation in the microminipig. In Vivo 27: 357-361, 2013.

29 Miyoshi K, Kawaguchi H, Maeda K, Sato M, Akioka K, Noguchi M, Horiuchi $\mathrm{M}$ and Tanimoto A: Birth of cloned microminipigs derived from somatic cell nuclear transfer embryos that have been transiently treated with valproic acid. Cell Reprogram 18: 390-400, 2016.

30 Yamada S, Kawaguchi H, Yamada T, Guo X, Matsuo K, Hamada $\mathrm{T}$, Miura N, Tasaki $\mathrm{T}$ and Tanimoto A: Cholic acid enhances visceral adiposity, atherosclerosis and nonalcoholic fatty liver disease in microminipigs. J Atheroscler Thromb 24: 1150-1166, 2017.
31 Vaughn DM, Coleman E, Simpson ST, Whitmer B and Satjawatcharaphong C: A rostrocaudal gradient for neurotransmitter metabolites and a caudorostral gradient for protein in canine cerebrospinal fluid. Am J Vet Res 49: 2134-2137, 1988.

32 Djukic M, Spreer A, Lange P, Bunkowski S, Wiltfang J and Nau R: Small cisterno-lumbar gradient of phosphorylated Tau protein in geriatric patients with suspected normal pressure hydrocephalus. Fluids Barriers CNS 13: 15, 2016.

33 Bruce J, Tamarkin L, Riedel C, Markey S and Oldfield E: Sequential cerebrospinal fluid and plasma sampling in humans: 24hour melatonin measurements in normal subjects and after peripheral sympathectomy. J Clin Endocrinol Metab 72: 819-823, 1991.

34 Tan D-X, Manchester LC and Reiter RJ: CSF generation by pineal gland results in a robust melatonin circadian rhythm in the third ventricle as a unique light/dark signal. Med Hypotheses 86: 3-9, 2016.
Received February 17, 2018

Revised March 10, 2018

Accepted March 12, 2018 